\title{
Temporal trends of bladder cancer incidence and mortality from 1990 to 2016 and projections to 2030
}

\author{
Qiliang Cai ${ }^{1,2 \#}$, Yegang Chen ${ }^{1 \#}$, Shuhui Xin ${ }^{3}$, Dingrong Zhang ${ }^{1}$, Jiancheng Pan ${ }^{1}, Z_{\text {Zunk Xie }}{ }^{1}$, Chenjie Xu ${ }^{2}$, \\ Shu $\mathrm{Li}^{2}$, Xinyu Zhang ${ }^{2}$, Ying Gao ${ }^{4}$, Jie Hou ${ }^{5}$, Xuemei Guo ${ }^{6}$, Xiaodong Zhou ${ }^{1}$, Baoshuai Zhang ${ }^{7}, \mathrm{Fei} \mathrm{Ma}^{8}$, \\ Wei Zhang ${ }^{1}$, Guiting Lin', Zhongcheng Xin ${ }^{1,10}$, Yuanjie Niu', Yaogang Wang ${ }^{2}$
}

${ }^{1}$ Department of Urology, the Second Hospital of Tianjin Medical University, Tianjin Institute of Urology, Tianjin 300211, China; ${ }^{2}$ School of Public Health, Tianjin Medical University, Tianjin 300070, China; ${ }^{3}$ The Second Hospital of Tianjin Medical University, Tianjin 300211, China; ${ }^{4}$ Department of Health Management, Tianjin Medical University General Hospital, Tianjin 300052, China; ${ }^{5}$ School of Basic Medical Sciences, ${ }^{6}$ Library of Tianjin Medical University, Tianjin Medical University, Tianjin 300070, China; ${ }^{7}$ Scientific Research Department, the Second Hospital of Tianjin Medical University, Tianjin 300070, China; ${ }^{8}$ Department of Epidemiology and Biostatistics, School of Public Health, Tianjin Medical University, Tianjin 300070, China; ${ }^{9}$ Knuppe Molecular Urology Laboratory, Department of Urology, School of Medicine, University of California, San Francisco, CA, USA; ${ }^{10}$ Andrology Center, Peking University First Hospital, Peking University, Beijing 100034, China

Contributions: (I) Conception and design: Q Cai, Y Chen, Z Xin, Y Niu, Y Wang; (II) Administrative support: X Zhou, B Zhang, F Ma, W Zhang, G Lin; (III) Provision of study materials or patients: Q Cai, Y Chen, S Xin, D Zhang, J Pan; (IV) Collection and assembly of data: S Xin, D Zhang, J Pan, Z Xie, C Xu, S Li, X Zhang, Y Gao, J Hou, X Guo; (V) Data analysis and interpretation: Q Cai, Y Chen, S Xin, D Zhang, J Pan; (VI) Manuscript writing: All authors; (VII) Final approval of manuscript: All authors.

\#These authors contributed equally to this work.

Correspondence to: Prof. Yuanjie Niu. Department of Urology, the Second Hospital of Tianjin Medical University, Tianjin Institute of Urology, Tianjin 300211, China. Email: qlcwwwtg@gmail.com; Prof. Yaogang Wang. School of Public Health, Tianjin Medical University, Tianjin 300070, China. Email: wyg@tmu.edu.cn.

Background: Bladder cancer is a leading cause of cancer-related deaths all over the world. Epidemiological studies of bladder cancer are therefore crucial for policy making. This study was carried out to describe the characteristics of changes in the incidence and mortality of bladder cancer from 1990 to 2016 by age group, gender, geographical region, and sociodemographic index (SDI) and to simultaneously project future trends up to 2030.

Methods: Incidence and mortality trends in bladder cancer from 1990 to 2016 were described based on data and methodologies from the Global Burden of Disease (GBD) Study. The data also allowed the future trends of bladder cancer incidence and mortality to be predicted by ARIMA model. Trends were analyzed by age group, gender, and SDI. Projections to 2030 were sub-analyzed by SDI countries. R software (x64 version 3.5.1), SAS (version 9.3), and SPSS (version 22.0) were used throughout the process.

Results: Globally, in 2016, there were 437,442 [95\% uncertainty interval (UI), 426,709-447,912] new bladder cancer cases and 186,199 (95\% UI, 180,453-191,686) bladder cancer-associated deaths. Between 1990 and 2016, changes in the age-standardized incidence rate (ASIR) of bladder cancer decreased by 5.91\% from 7.11 (95\% UI, 6.93-7.27) in 1990 to 6.69 (95\% UI, 6.52-6.85) in 2016. The age-standardized death rate (ASDR) decreased from 3.58 (95\% UI, 3.49-3.68) to 2.94 (95\% UI, 2.85-3.03) over the same period of time. In future, the greatest occurrence of bladder cancer will be in high SDI countries, followed by highmiddle SDI countries. Moreover, bladder cancer incidence rates may increase substantially in middle SDI countries, while the incidence rates will remain relatively stable for men and women in other SDI countries. From 2017 to 2030, bladder cancer deaths will continue to increase in low SDI countries, while deaths in other SDI countries will continue to decrease.

Conclusions: There was a regional difference in the incidence and mortality trends of bladder cancer between 1990 and 2016. Overall, the situation is not optimistic. From 2017 to 2030, the incidence of bladder cancer will continue to rise, especially in high and high-middle SDI countries, where decision-makers should propose appropriate policies on the screening and prevention of bladder cancer. 
Keywords: Bladder cancer; incidence; mortality; over-time trends; projections

Submitted Jan 06, 2020. Accepted for publication Feb 14, 2020.

doi: $10.21037 /$ tau.2020.02.24

View this article at: http://dx.doi.org/10.21037/tau.2020.02.24

\section{Introduction}

Bladder cancer is one of the most common types of cancer and is a prominent cause of cancer-related deaths all over the world (1). Bladder cancer is more likely to affect older people and men (2), and along with the growing and ageing global population, the number of cancer-related deaths has dramatically increased, which in part can be attributed to its recurrence rate of up to $75 \%(1,3)$.

Due to international differences in the incidence and mortality of bladder cancer, the burden of bladder cancer is becoming more noticeable $(4,5)$. Necessary data to inform health policies with respect to urologic cancer at a global level, including cancer control and implementation plans, are not widely available. This study therefore aims to describe the global burden of urologic cancer from 1990 to 2016 according to age, sex, region, and sociodemographic index (SDI) (a summary indicator of income per capita, educational attainment, and fertility), and then forecast worldwide bladder cancer incidence and mortality rates for the next 15 years. Understanding these factors is critical for providing detailed information on bladder cancer etiologies and their trends over time, without which targeted prevention strategies are impossible to design and evaluate, and for promoting strategic investments into research and clinical resources.

\section{Methods}

Methodology and research processes for the Global Burden of Disease (GBD) Study have been reported previously in many publications (6-11). Herein, we present methods for the incidence, mortality, trends, and 15-year prediction of bladder cancer from 1990 to 2030 based on the data collected from the GBD study. Our study complies with the Guidelines for Accurate and Transparent Health Estimates Reporting (GATHER) and the detailed methodology also complies with the GATHER guidelines (12). Tables, which can be found in the Supplementary materials (Tables S1-S9), record the estimation steps and other detailed information about the methodology (7,13-15). The International
Classification of Diseases 10 (ICD-10) codes corresponding to bladder cancer incidence and mortality are C67-C67.9, Z12.6-Z12.79, Z80.52, Z85.51 and C67-C67.9, D09.0, D30.3, D41.4-D41.8, D49.4, respectively.

The GBD estimation process starts with cancer mortality. Data sources for bladder cancer mortality include vital registration systems and cancer registries. Cancer incidence data are used to model mortality in places where without cancer mortality data by multiplying incidence by a separately modelled mortality-to incidence ratio. These mortality estimates are used in a cause of death ensemble model $(\mathrm{CODEm})(7,11)$. Each cancer type is estimated separately using covariates with a causal connection. Cancer incidence is estimated by dividing final cancer-specific mortality estimates by the mortality-to-incidence ratio. We also estimated the contributions of population ageing, population growth, and the change in age-specific rates on the change of incident cases between 2006 and 2016, which are available in the Supplementary materials (6).

\section{Results}

Trends in bladder cancer incidence in the period from 1990 to 2016

Globally, new bladder cancer diagnoses rose 1.8-fold between 1990 and $2016\{238,617$ [95\% uncertainty interval (UI), 232,159-244,362] to 437,442 (95\% UI, 426,709$447,912)\}$. In 2016, in relation to SDI countries, the highest bladder cancer incidence in both men and women occurred in high SDI countries (213,500, 95\% UI, 206,159-220,038), followed by high-middle SDI countries $(96,865 ; 95 \%$ UI, 93,605-100,135), middle SDI countries (85,371; $95 \%$ UI, $80,381-87,836)$, low-middle SDI countries $(34,771 ; 95 \%$ UI, 32,913-36,384), and low SDI countries $(8,223 ; 95 \%$ UI, $7,850-8,585)$. In the same year, 334,373 (95\% UI, 325,085$342,168)$ and 103,069 (95\% UI, 98,687-107,068) incident cases were estimated for males and females, respectively. In terms of SDI countries, the largest increase $(162.60 \%)$ was in middle SDI countries [from 32,510 (95\% UI, $31,501-34,224)$ in 1990 to 85,371 (95\% UI, $80,381-87,836$ ) 
in 2016]. Among the regions, the largest increase in bladder cancer incident cases from 1990 to 2016 was in Oceania, with a rise of $259.52 \%$ [from 42 (95\% UI, 37-55) in 1990 to 151 (95\% UI, 133-185) in 2016]. In 2016, bladder cancer was more common in men, with 334,373 incident cases compared to women, with 103,069 cases (Table 1).

\section{Trends in bladder cancer-related deaths in the period from 1990 to 2016}

Globally, there were 186,199 (95\% UI, 180,453-191,686) deaths from bladder cancer in 2016, and the number of deaths was 137,883 (95\% UI, 133,054-142,437) in males and 48,315 (95\% UI, 46,028-50,487) in females. Between 1990 and 2016, deaths from bladder cancer rose 1.6-fold $[113,414(95 \%$ UI, $110,361-117,066)$ to $186,199(95 \%$ UI, 180,453-191,686)]. In terms of SDI countries, the highest burden of bladder cancer deaths for both men and women occurred in high SDI countries $(75,336$; 95\% UI, 72,679-77,841), followed by middle SDI countries (41,248; 95\% UI, 38,787-42,863), high-middle SDI countries (38,889; $95 \%$ UI, 36,379-41,379), low-middle SDI countries $(22,311 ; 95 \%$ UI, 20,831-23,545), and low SDI countries $(8,320 ; 95 \%$ UI, 7,757-8,853). However, there was a decrease $(18.65 \%)$ in bladder cancer deaths observed across all SDI countries from 1990 to 2016, especially in high-middle SDI countries. Among the regions, Oceania experienced the largest increase in bladder cancer-related deaths from 1990 to 2016, with a rise of $14.92 \%$ [from 29 (95\% UI, 24-38) in 1990 to 78 (95\% UI, 64-103) in 2016]. In 2016, bladder cancer mortality was much higher in men compared to women, with the number of death cases standing at 137,883 and 48,315, respectively (Table 1).

\section{Trends in the age-standardized incidence rate (ASIR) of bladder cancer in the period from 1990 to 2016}

Globally, between 1990 and 2016, changes in the ASIR for bladder cancer decreased by $5.91 \%$ from $7.11(95 \%$ UI, 6.93-7.27) in 1990 to 6.69 (95\% UI, 6.52-6.85) in 2016. In 2016, the highest ASIR was in high SDI countries (11.92; 95\% UI, 11.53-12.28). However, the ASIR of bladder cancer in both sexes decreased in high SDI, highmiddle SDI, and low SDI countries while it increased in middle SDI and low-middle SDI countries from 1990 to 2016. Between 1990 and 2016, ASIRs of bladder cancers remained highest in Western Europe, where the ASIR decreased by $7.97 \%$ from 15.93 (95\% UI, 15.57-16.30) to
14.66 (95\% UI, 13.93-15.24), followed by Central Europe, where it rose from 9.23 (95\% UI, 9.03-9.45) to 12.05 (95\% UI, 11.62-12.51). Globally, the age-standard bladder cancer incidence rate (per 100,000 people) in 2016 among men $[11.54(11.21-11.81)]$ was 4 times higher than among women [2.86 (2.75-2.98)] (Table 2, Figures 1,2).

\section{Trends in the age-standardized death rate (ASDR) of bladder cancer in the period from 1990 to 2016}

Globally, the ASDR of bladder cancer decreased from 3.58 (95\% UI, 3.49-3.68) in 1990 to 2.94 (95\% UI, 2.85-3.03) in 2016. Between 1990 and 2016, changes in ASDRs was highest among men in low-middle SDI countries, where the ASDRs increased by 4.58\% from 2.84 (95\% UI, 2.45-3.14) to 2.97 (95\% UI, 2.71-3.21). Between 1990 and 2016, in terms of region, the highest changes in ASDR occurred in Oceania, followed by Central Europe. Simultaneously, ASDR decreased substantially in East Asia and Southern Latin America. Globally, the mortality rates of bladder cancer were higher among males than females, with male-tofemale ratios of 3.7:1, which indicated that the age-standard bladder cancer death rate (per 100,000 people) among men [5.10 (4.93-5.27)] was approximately 3.7-fold higher than among women [1.35 (1.28-1.41)] (Table 2, Figures 2,3).

\section{Projections of bladder cancer incidence and mortality from 2017 to 2030}

This study also forecast the trends in the incidence and mortality rates of bladder cancer from 2017 to 2030. Bladder cancer will occur most frequently in high SDI countries, followed by high-middle SDI countries. Bladder cancer incidence rates may increase substantially in middle SDI countries, while incidence rates for both men and women should remain relatively stable in other SDI countries. Deaths due to bladder cancer will continue to increase in low SDI countries, while in other SDI countries the decrease from 1990 will continue to 2030 . Globally, the probability of developing bladder cancer for men between the ages of 30 and 70 is around $1.32 \%$, compared to $0.32 \%$ for women. In other words, the estimated risk of suffering from bladder cancer is higher among men than women. Similar results can be seen in other age intervals and SDI countries (Table 3, Figure 4).

\section{Discussion}

The estimates presented in this study reveal remarkable 
Table 1 Global and regional bladder cancer incident and death cases by geography, gender, and SDI, 1990 and 2016

\begin{tabular}{|c|c|c|c|c|c|c|c|c|c|c|c|c|}
\hline \multirow{3}{*}{ Location } & \multicolumn{6}{|c|}{ Incident cases, global and regional } & \multicolumn{6}{|c|}{ Death cases, global and regional } \\
\hline & \multicolumn{3}{|c|}{1990} & \multicolumn{3}{|c|}{2016} & \multicolumn{3}{|c|}{1990} & \multicolumn{3}{|c|}{2016} \\
\hline & Male & Female & Both & Male & Female & Both & Male & Female & Both & Male & Female & Both \\
\hline Global & $179,438[174,049-184,282]$ & $59,179[57,539-60,890]$ & $238,617[232,159-244,362]$ & $334,373[325,085-342,168]$ & $103,069[98,687-107,068]$ & $437,442[4266,709-447,912]$ & $81,981[79,064-85,004]$ & $31,433[30,267-32,783]$ & $113,414[110,361-117,066]$ & $137,883[133,054-142,437]$ & $48,315[46,028-50,487]$ & $186,199[180,453-191,686]$ \\
\hline High SDI & $101,014[99,063-103,041]$ & $32,979[32,367-33,606]$ & $133,992[131,968-136,064]$ & $161,396[154,512-166,577]$ & $52,104[49,679-54,630]$ & $213,500[206,159-220,038]$ & $35,703[35,030-36,370]$ & $14,346[14,031-14,669]$ & $50,049[49,298-50,885]$ & $54,949[52,530-57,085]$ & $20,387[19,284-21,390]$ & $75,336[72,679-77,841]$ \\
\hline High-middle SDI & $43,298[39,889-45,673]$ & $11,490[10,833-12,202]$ & $54,788[[51,061-57,281]$ & $77,144[74,433-79,912]$ & $19,721[18,543-20,826]$ & $96,865[93,605-100,135]$ & $20,432[18,799-21,725]$ & $5,790[5,482-6,115]$ & $26,223[24,462-27,545]$ & $30,484[28,212-32,743]$ & $8,404[7,598-9,352]$ & $38,889[36,379-41,379]$ \\
\hline Low SDI & $2,512[2,361-2,905]$ & $1,513[1,303-1,797]$ & $4,025[3,709-4,547]$ & $5,193[4,866-5,488]$ & 3,030 [2,848-3,239] & $8,223[7,850-8,585]$ & $2,489[2,236-2,930]$ & $1,510[1,286-1,809]$ & $3,999[3,631-4,545]$ & $5,274[4,773-5,712]$ & $3,046[2,723-3,346]$ & $8,320[7,757-8,853]$ \\
\hline Low-middle SDI & $9,893[8,765-10,706]$ & $4,498[4,029-5,279]$ & $14,391[12,928-15,622]$ & $25,581[23,840-27,099]$ & $9,190[8,856-9,530]$ & $34,771[32,913-36,384]$ & $7,189[6,256-7,946]$ & $3,662[3,082-4,402]$ & $10,851[9,872-11,959]$ & $15,795[[4,474-17,080]$ & $6,516[5,972-7,038]$ & $22,311[20,831-23,545]$ \\
\hline Middle SDI & $23,751[23,023-25,145]$ & $8,759[8,342-9,217]$ & $32,510[31,501-34,224]$ & $66,277[62,215-68,205]$ & $19,094[17,794-20,156]$ & $85,371[80,381-87,836]$ & $16,144[15,408-17,259]$ & $6,111[5,744-6,443]$ & $22,254[21,368-23,483]$ & $31,314[29,333-32,668]$ & $9,934[9,182-10,567]$ & $41,248[38,787-42,863]$ \\
\hline High-income Asia Pacific & $8,994[8,746-9,250]$ & $3,114[3,027-3,197]$ & $12,108[11,837-12,374]$ & $21,513[19,918-22,754]$ & $7,581[7,129-8,098]$ & $29,094[27,312-30,653]$ & $2,875[2,781-2,978]$ & $1,308[1,266-1,355]$ & $4,183[4,080-4,303]$ & $7,038[6,440-7,622]$ & 3,030 [2,793-3,291] & $10,067[9,365-10,749]$ \\
\hline Andean Latin & 269 [253-289] & $142[126-160]$ & $411[385-443]$ & $622[581-667]$ & 356 [331-381] & $978[935-1,023]$ & 189 [172-206] & $100[87-116]$ & $288[266-315]$ & 391 [337-452] & $225[$ [189-265] & $616[550-687]$ \\
\hline America & $1,221[1,184-1,260]$ & 644 [624-666] & $1,865[1,818-1,910]$ & $3,644[3,539-3,759]$ & $1,995[1,871-2,072]$ & $5,639[5,494-5,778]$ & $785[758-814]$ & $420[4044-437]$ & $1,205[1,172-1,239]$ & $1,769[1,673-1,873]$ & $977[904-1,038]$ & $2,747[2,624-2,870]$ \\
\hline Central Latin America & $2,412[2,274-2,557]$ & 624 [581-669] & $3,036[2,887-3,192]$ & $3,901[3,619-4,187]$ & $1,466[1,346-1,596]$ & $5,367[5,050-5,682]$ & $1,370[1,272-1,467]$ & $397[364-431]$ & $1,766[1,664-1,869]$ & $1,781[1,577-1,992]$ & $762[662-874]$ & $2,543[2,317-2,792]$ \\
\hline Southern Latin America & $2,131[2,058-2,204]$ & $820[786-855]$ & $2,952[2,869-3,038]$ & $6,311[6,047-6,574]$ & $2,942[2,776-3,100]$ & $9,253[8,945-9,559]$ & $1,334[1,273-1,399]$ & $519[4933-546]$ & $1,853[1,786-1,924]$ & $3,234[3,034-3,427]$ & $1,482[1,375-1,594]$ & $4,715[4,490-4,947]$ \\
\hline Tropical Latin America & $7,453[6,903-8,204]$ & $1,864[1,661-2,086]]$ & $9,316[8,680-10,212]$ & $22,984[21,176-24,621]$ & $5,142[4,743-5,619]$ & $28,126[26,065-30,002]$ & $5,002[4,479-5,615]$ & $1,268[1,106-1,454]$ & $6,270[5,624-6,986]$ & $10,655[9,643-11,721]$ & $2,496[2,278-2,775]$ & $13,152[12,143-14,214]$ \\
\hline North Africa and Middle & $27,248[26,618-27,875]$ & $10,465[10,158-10,789]$ & $37,712[33,932-38,481]$ & $46,158[44,662-47,633]$ & $15,905[15,200-16,620]$ & $62,062[60,383-63,707]$ & $8,929[8,698-9,161]$ & $4,046[3,921-4,175]$ & $12,975[[12,688-13,262]$ & $15,028[[14,427-15,637]$ & $5,891[5,605-6,216]$ & $20,919[20,235-21,653]$ \\
\hline East & 27 [25-36] & 15 [12-20] & $42[37-55]$ & 97 [88-119] & 54 [42-70] & 151 [133-185] & 18 [15-24] & $11[8-16]$ & 29 [24-38] & $47[40-63]$ & $32[23-44]$ & 78 [64-103] \\
\hline High-income North America & 375 [324-464] & 238 [197-290] & $613[533-718]$ & $744[676-815]$ & 432 [381-490] & $1,176[1,100-1,256]$ & 340 [271-428] & 219 [179-273] & 559 [465-662] & 705 [605-820] & $424[363-487]$ & $1,130[1,009-1,264]$ \\
\hline Oceania & $1,050[975-1,146]$ & $809[$ [699-942] & $1,859[1,725-1,999]$ & $2,335[2,060-2,538]$ & $1,758[1,479-2,089]$ & $4,094[3,567-4,514]]$ & $1,069[954-1,203]$ & $835[692-1,002]$ & $1,904[1,722-2,108]$ & $2,375[2,036-2,656]$ & $1,739[1,411-2,077]$ & $4,115[3,529-4,533]$ \\
\hline Eastern sub-Saharan & $1,199[1,140-1,280]$ & 558 [513-604] & $1,757[1,670-1,872]$ & $2,163[2,060-2,256]$ & $967[869-1,052]$ & $3,129[2,959-3,268]$ & $1,206[1,082-1,343]$ & $583[516-662]$ & $1,789[1,630-1,961]$ & $2,120[1,922-2,362]$ & $941[811-1,081]$ & $3,061[2,794-3,363]$ \\
\hline Africa & $18,715[17,988-19,829]$ & $6,821[6,436-7,230]$ & $25,536[24,567-26,906]$ & $51,759[48,743-53,797]$ & $12,867[11,731-14,081]$ & $64,626[60,665-67,499]$ & $12,258[11,642-13,087]$ & $4,756[4,386-5,105]$ & $17,014[16,205-17,924]$ & $21,531[[20,229-22,538]$ & $6,070[5,517-6,665]$ & $27,601[25,919-28,929]$ \\
\hline Central Asia & $8,119[7,279-8,781]$ & $3,215[2,891-3,748]$ & $11,334[11,216-12,246]$ & $22,160[20,533-23,458]$ & $7,281[[6,918-7,677]$ & $29,440[27,591-30,984]$ & $5,561[4,829-6,166]$ & $2,375[1,996-2,844]$ & $7,936[7,126-8,737]$ & $13,054[[1,845-14,181]$ & $4,820[4,303-5,287]$ & $17,874[16,426-19,107]$ \\
\hline Southern sub-Saharan & $3,862[3,634-4,317]$ & $1,802[1,501-2,162]$ & $5,664[5,185-6,330]$ & $11,956[11,335-12,490]$ & $4,117[3,818-4,503]$ & $16,073[[15,403-16,759]$ & $2,619[2,394-2,264]]$ & $1,463[1,178-1,830]$ & $4,082[3,661-4,598]$ & $5,869[5,460-6,291]$ & $2,571[2,336-2,885]$ & $8,440[7,944-9,008]$ \\
\hline Africa & $1,881[1,774-1,996]$ & 638 [591-684] & $2,519[2,402-2,642]$ & $3,008[2,754-3,289]$ & $1,018[916-1,127]$ & $4,026[3,755-4,333]$ & $617[579-657]$ & $267[248-287]$ & 884 [845-927] & $1,050[937-1,170]$ & $432[380-495]$ & $1,482[1,361-1,618]$ \\
\hline Western sub-Saharan & $845[803-892]$ & $342[314-376]$ & $1,186[1,131-1,253]$ & $1,742[1,643-1,845]$ & $718[664-775]$ & $2,460[2,346-2,576]$ & $477[446-513]$ & 203 [183-230] & $681[638-732]$ & $833[758-908]$ & $365[325-410]$ & $1,198[1,117-1,285]$ \\
\hline Africa & $9,479[9,189-9,769]$ & $2,796[2,688-2,906]$ & $12,276[11,983-12,579]$ & $17,011[16,318-17,768]$ & $5,495[5,135-5,878]$ & $22,506[21,692-23,381]$ & $4,709[4,517-4,881]$ & $1,391[1,334-1,452]$ & $6,100[5,898-6,277]$ & $7,202[6,774-7,709]$ & 2,295[2,114-2,488] & $9,496[9,033-10,048]$ \\
\hline East Asia & $17,461[14,748-19,251]$ & $4,904[4,373-5,519]$ & $22,365[19,368-24,346]$ & $20,295[18,847-21,796]$ & $5,549[5,091-6,053]$ & $25,844[24,301-27,409]$ & $7,882[[6,603-8,865]$ & $2,063[1,794-2,366]$ & $9,945[8,562-11,004]$ & $8,885[6,996-10,985]$ & $2,285[1,680-3,046]$ & $11,169[9,211-13,423]$ \\
\hline
\end{tabular}


Table 2 Global and regional age-standardized bladder cancer incidence and death rates with $95 \%$ uncertainty interval and percent change by geography, gender, and SDI between 1990 and 2016

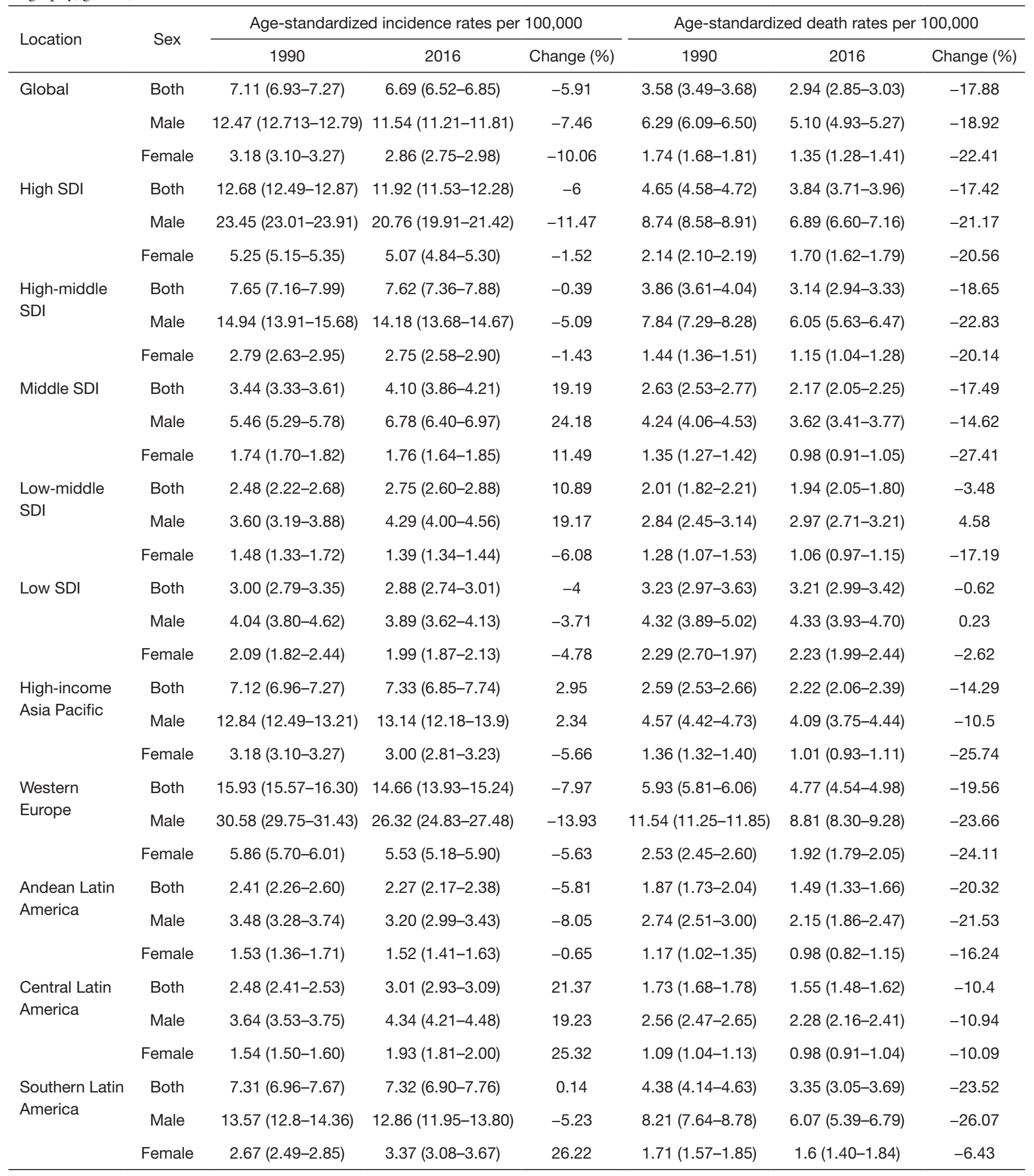

Table 2 (continued) 
Table 2 (continued)

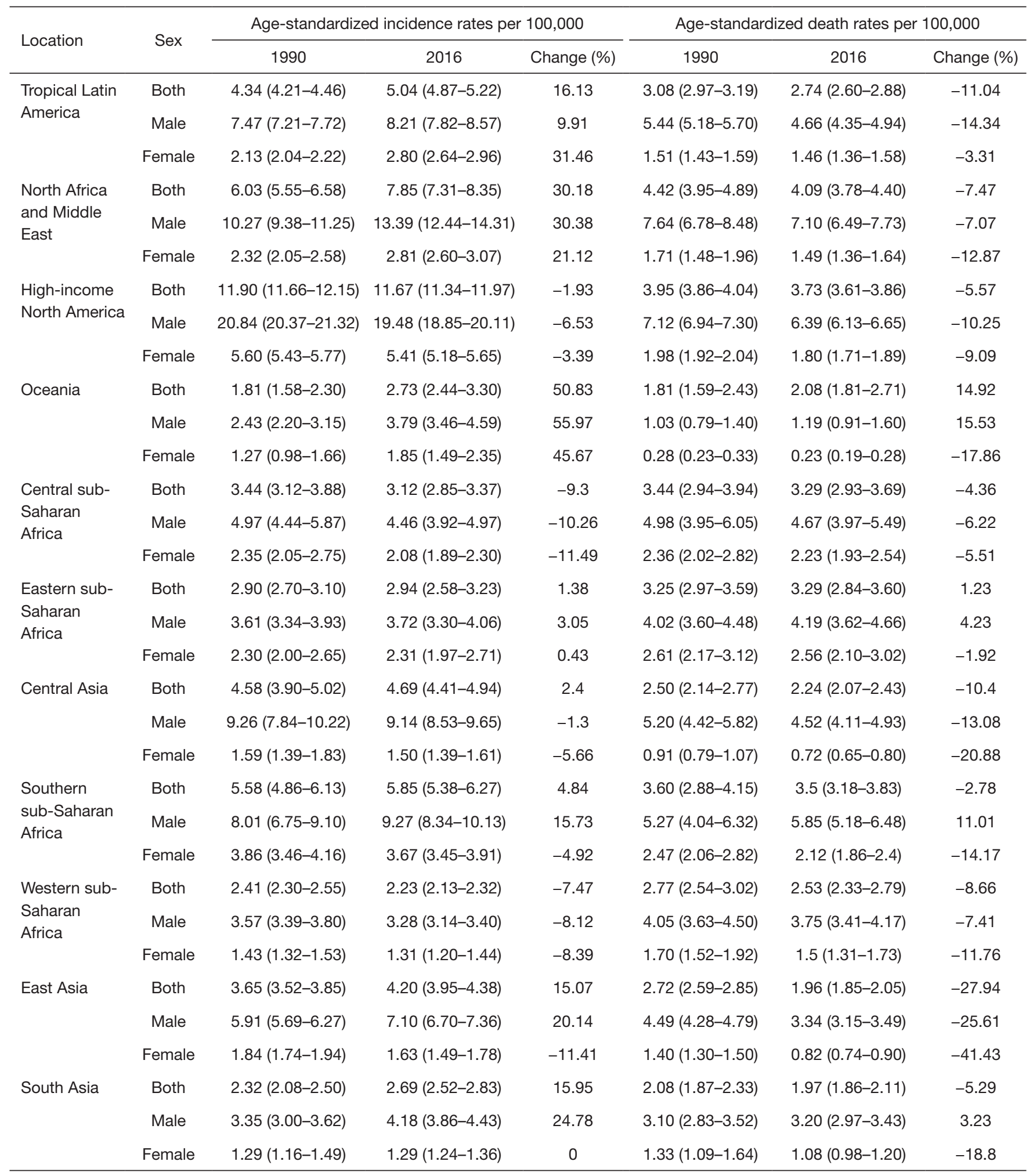

Table 2 (continued) 
Table 2 (continued)

\begin{tabular}{|c|c|c|c|c|c|c|c|}
\hline Location & Sex & \multicolumn{3}{|c|}{ Age-standardized incidence rates per 100,000} & \multicolumn{3}{|c|}{ Age-standardized death rates per 100,000} \\
\hline \multirow{3}{*}{$\begin{array}{l}\text { Southeast } \\
\text { Asia }\end{array}$} & Both & $2.63(2.42-2.93)$ & $3.34(3.21-3.48)$ & 27 & $2.08(1.87-2.33)$ & $1.97(1.86-2.11)$ & -5.29 \\
\hline & Male & $4.07(3.83-4.56)$ & $5.59(5.31-5.84)$ & 37.35 & $3.10(2.83-3.52)$ & $3.20(2.97-3.43)$ & 3.23 \\
\hline & Female & $1.52(1.29-1.80)$ & $1.58(1.47-1.72)$ & 3.95 & $1.33(1.09-1.64)$ & $1.08(0.98-1.20)$ & -18.8 \\
\hline \multirow{2}{*}{ Australasia } & Male & $20.58(19.50-21.78)$ & $15.54(14.23-16.95)$ & -24.49 & $7.31(6.88-7.76)$ & $5.33(4.78-5.92)$ & -27.09 \\
\hline & Female & $5.27(4.90-5.65)$ & $4.35(3.92-4.80)$ & -17.46 & $2.13(1.97-2.28)$ & $1.64(1.44-1.88)$ & -23 \\
\hline \multirow[t]{2}{*}{ Caribbean } & Both & $5.08(4.84-5.37)$ & $5.61(5.35-5.89)$ & 10.43 & $3.00(2.82-3.22)$ & $2.74(2.56-2.94)$ & -8.67 \\
\hline & Male & 7.76 (7.37-8.19) & $8.76(8.26-9.27)$ & 12.89 & $4.58(4.29-4.90)$ & $4.32(3.93-4.71)$ & -5.68 \\
\hline \multirow{2}{*}{$\begin{array}{l}\text { Central } \\
\text { Europe }\end{array}$} & Male & $17.08(16.59-17.55)$ & $21.60(20.72-22.52)$ & 26.46 & $9.05(8.70-9.36)$ & $9.37(8.81-10.01)$ & 6.94 \\
\hline & Female & 3.68 (3.55-3.81) & $5.16(4.82-5.51)$ & 40.22 & $1.83(1.76-1.91)$ & $1.94(1.79-2.1)$ & 9.95 \\
\hline \multirow{3}{*}{$\begin{array}{l}\text { Eastern } \\
\text { Europe }\end{array}$} & Both & $8.84(7.70-9.60)$ & $8.31(7.84-8.80)$ & -6 & $4.02(3.49-4.42)$ & $3.53(2.90-4.25)$ & -12.19 \\
\hline & Male & $20.46(17.59-22.39)$ & 18.04 (16.84-19.34) & -11.83 & $10.34(8.86-11.50)$ & $8.37(6.68-10.22)$ & -19.05 \\
\hline & Female & $3.06(2.73-3.45)$ & $2.85(2.62-3.09)$ & -6.86 & $1.26(1.09-1.43)$ & $1.08(0.79-1.44)$ & -14.29 \\
\hline
\end{tabular}

Data in the parentheses indicates $95 \%$ uncertainty interval (95\% UI). SDI, Sociodemographic index (a summary indicator of income per capita, educational attainment, and fertility).

differences in trends in the incidence and death rates of bladder cancer from 1990 to 2016. New bladder cancer diagnoses and related deaths increased while the ASIR and ASDR decreased over the same time period. At a global level, between 1990 and 2016, the decreasing incidence rate of bladder cancer was particularly noticeable. Cancer outcomes depend on health care expenditures as well as adequate diagnosis and treatment services (11). Trends in the incidence and death rates of bladder cancer were evaluated for both sexes, and these rates were observed to be greater in males than females across all SDI countries and most regions. A large proportion of the increase in bladder cancer incidence can be attributed to improving life expectancy and population growth (16). Tobacco smoking is the dominant risk factor for bladder cancer and is associated with $50-65 \%$ of male cases and $20-30 \%$ of female cases (17-19). Furthermore, genetic predisposition, occupational exposure to aromatic amines, polycyclic aromatic hydrocarbons, chlorinated hydrocarbons, cyclophosphamide, pioglitazone, exposure to ionising radiation, and chlorination of drinking water and subsequent levels of trihalomethanes have all been suggested as contributing to the development of bladder cancer (20). Clinically, early symptoms of bladder cancer include bloody urine or pain during urination. In its early stages, the disease can be treated cystoscopically plus immunotherapy with Bacillus Calmette-Guérin (BCG) (21); however, up to $70 \%$ of tumors recur $(22,23)$. Early detection of bladder cancer and a high recurrence rate of bladder cancer may be two of the reasons for the sharp increase in the incidence and deaths of bladder cancer seen in recent years.

For chronic diseases such as bladder cancer, it is especially important to develop appropriate and reasonable strategies for prevention and control. Epidemiology references are absolutely imperative in bladder cancer prevention, screening, and treatment. In our study, data of bladder cancer were analyzed by age group, gender, geographical region, and SDI. We found that there are differences in the incidence and mortality of bladder cancer regardless of age group, gender, region, or SDI. Additionally, we also forecast the trends in the incidence and mortality rates of bladder cancer from 2017 to 2030. What we did has considerable significance in terms of the proposal of bladder cancer 
A
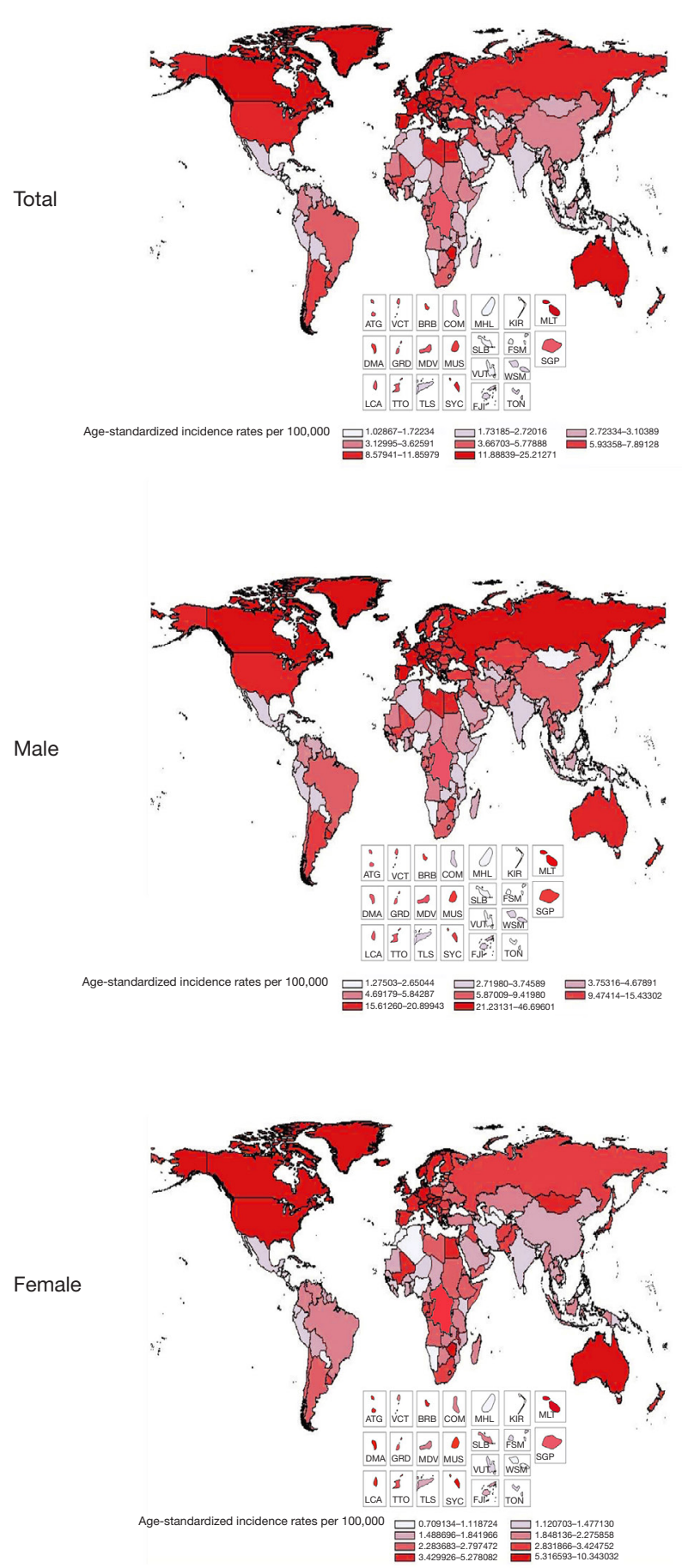

B

2016
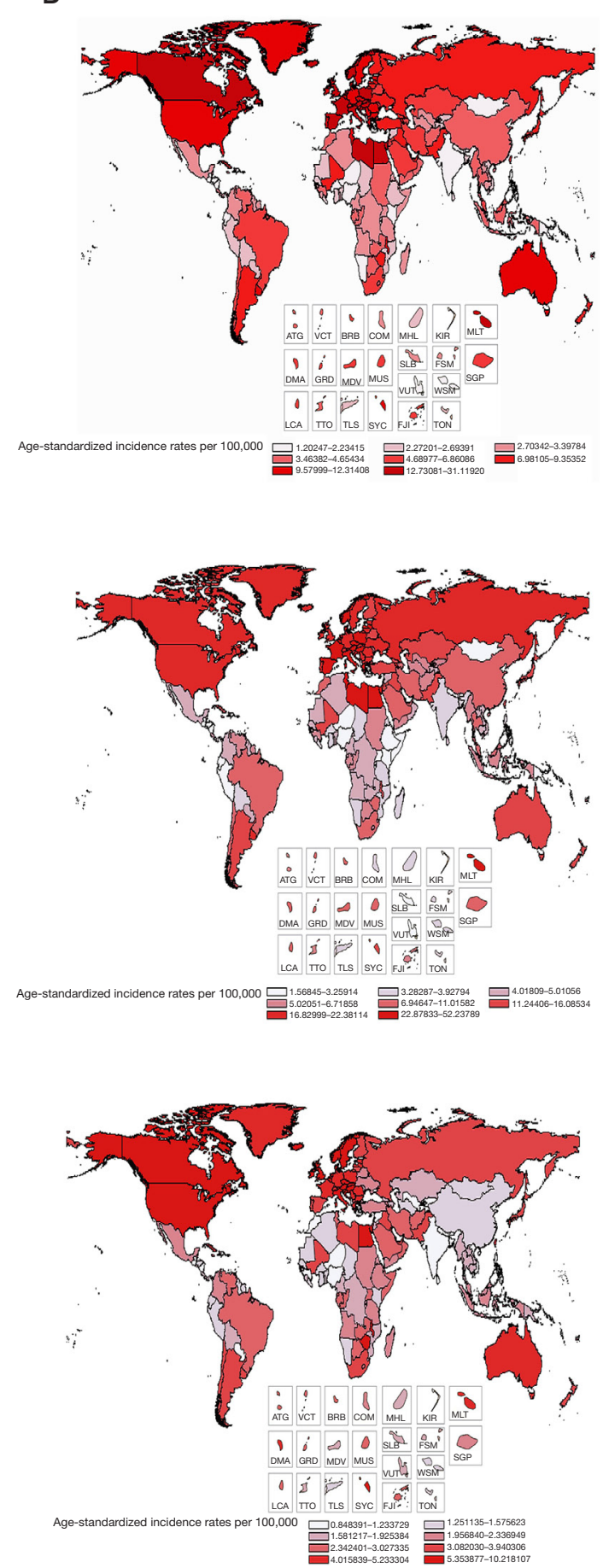

Figure 1 Global and regional bladder cancer ASIR by geography and gender, 1990 and 2016. ASIR, age-standardized incidence rate; ATG, Antigua and Barbuda; VCT, Saint Vincent and the Grenadines; BRB, Barbados; COM, Comoros; MHL, Marshall Islands; KIR, Kiribati; MLT, Malta; DMA, Dominica; GRD, Grenada; MDV, Maldives; MUS, Mauritius; SLB, Solomon Islands; FSM, Federated States of Micronesia; VUT, Vanuatu; WSM, Samoa. SGP, Singapore; LCA, Saint Lucia; TTO, Trinidad and Tobago; TLS, Timor-Leste; SYC, Seychelles; FJI, Fiji; TON, Tonga. 
A
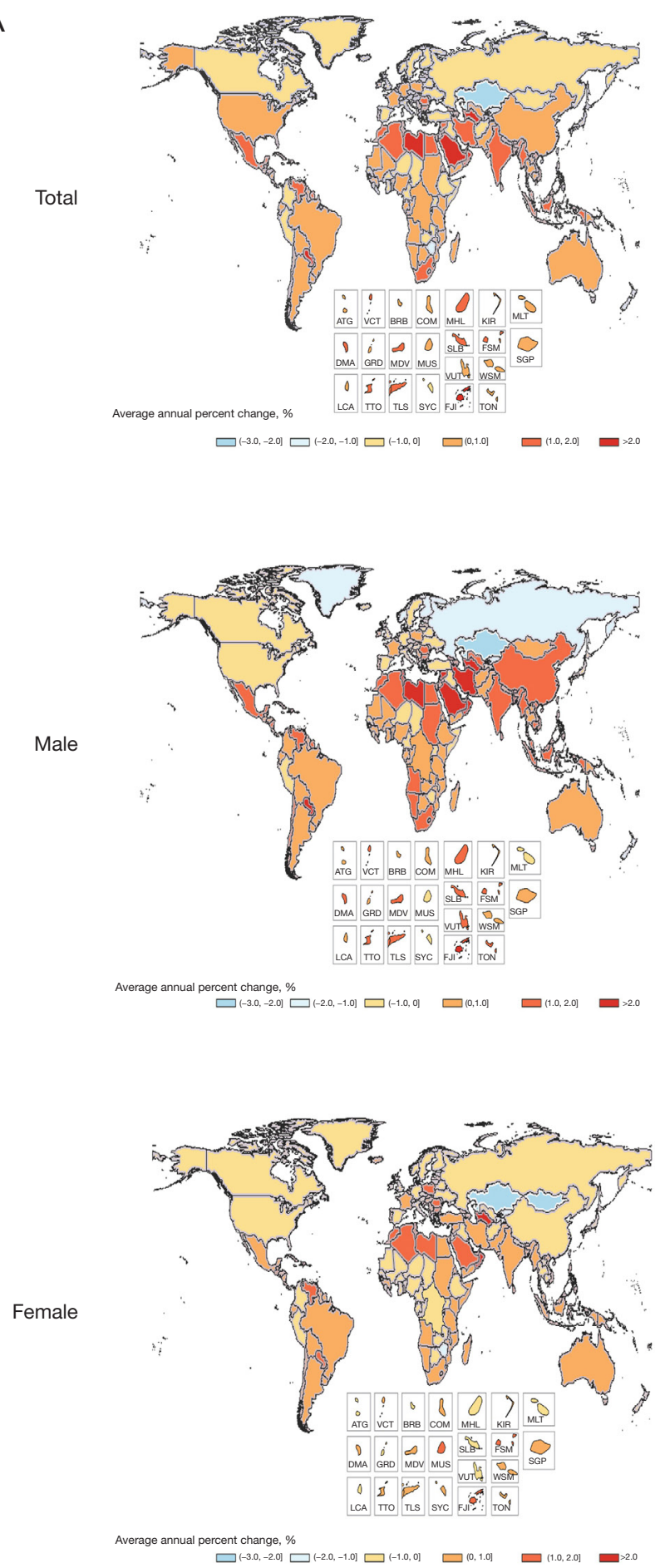

B

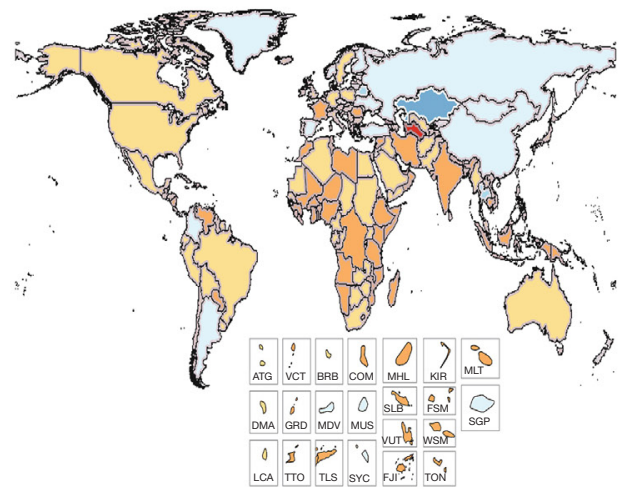

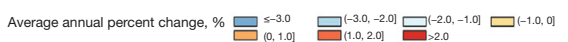

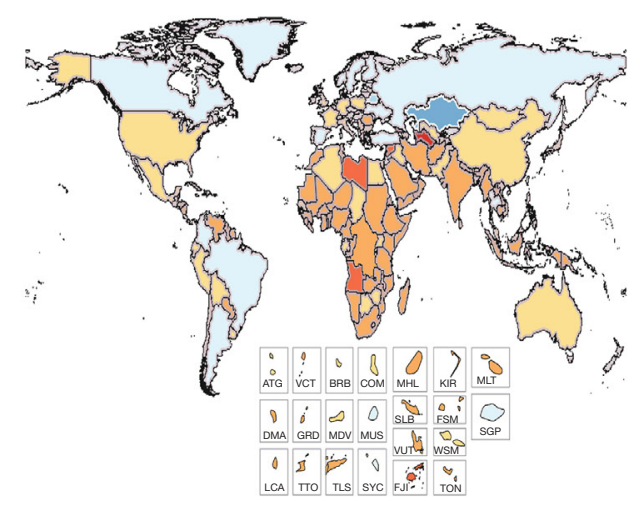

Average annual percent change, \% $\square_{[0,1.0]}^{s-3.0} \square^{(-3.0 .0,-2.0]} \square_{>2.0}^{(-2.0,-1.0]} \square^{(-1.0,0,0]}$

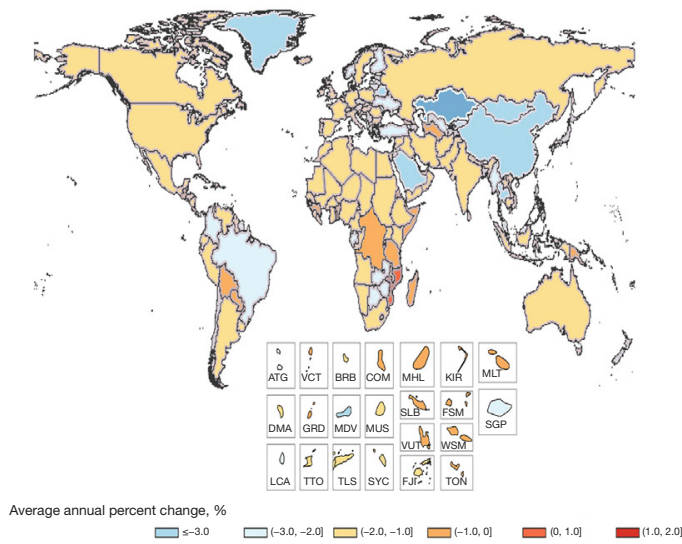

Figure 2 Global and regional average annual percent change in age-standardized incidence and death rates for bladder cancer by geography and gender, 1990-2016. (A) Average annual percent change in age-standardized incidence rates for bladder cancer by geography and gender, 1990-2016; (B) average annual percent change in age-standardized death rates for bladder cancer by geography and gender, 1990-2016. ATG, Antigua and Barbuda; VCT, Saint Vincent and the Grenadines; BRB, Barbados; COM, Comoros; MHL, Marshall Islands; KIR, Kiribati; MLT, Malta; DMA, Dominica; GRD, Grenada; MDV, Maldives; MUS, Mauritius; SLB, Solomon Islands; FSM, Federated States of Micronesia; VUT, Vanuatu; WSM, Samoa. SGP, Singapore; LCA, Saint Lucia; TTO, Trinidad and Tobago; TLS, Timor-Leste; SYC, Seychelles; FJI, Fiji; TON, Tonga. 
A

1990
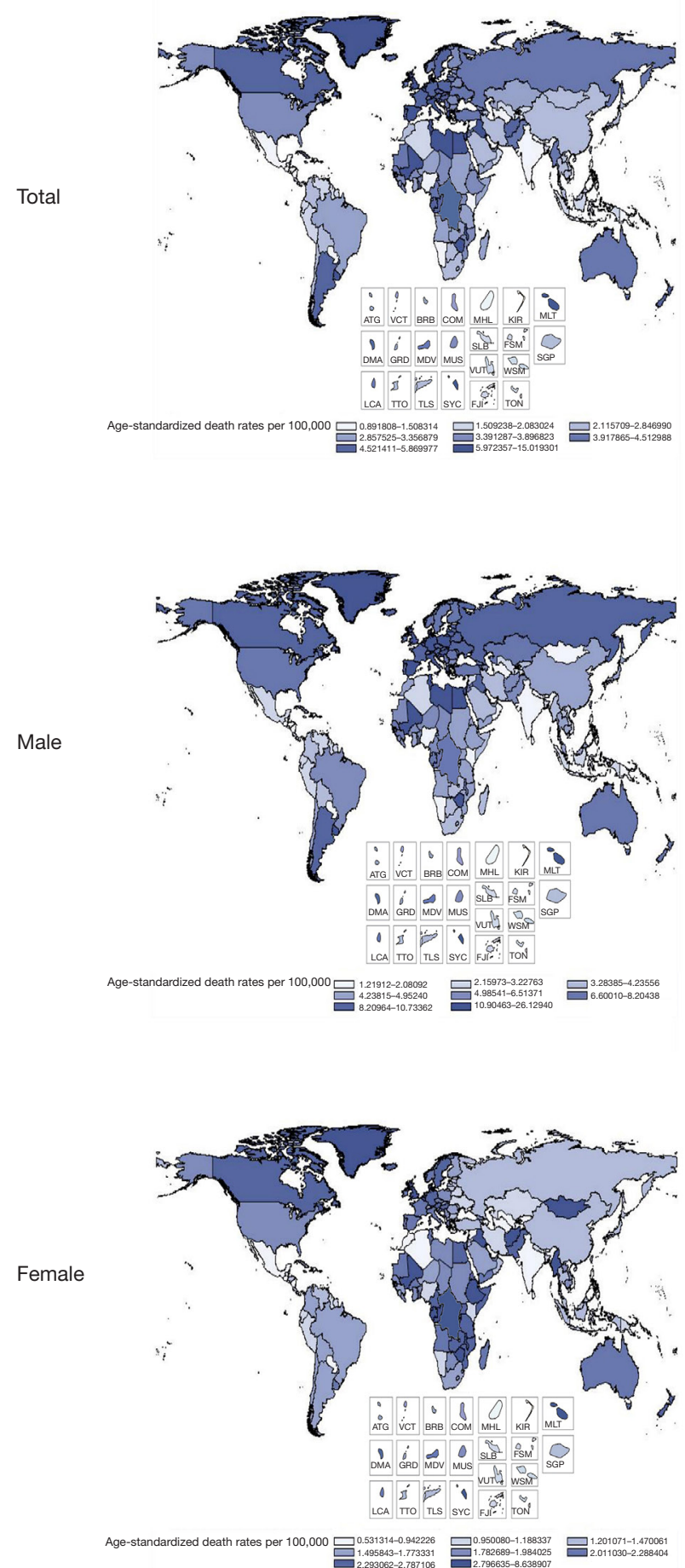

B
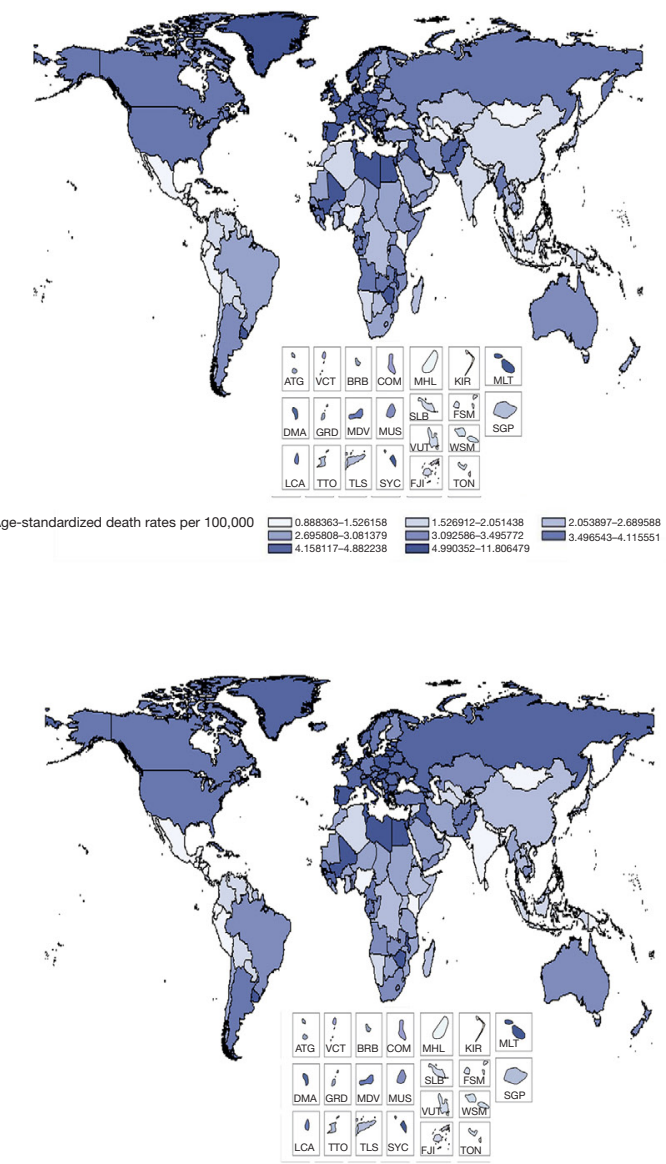

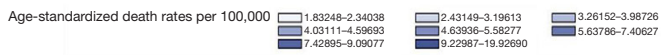

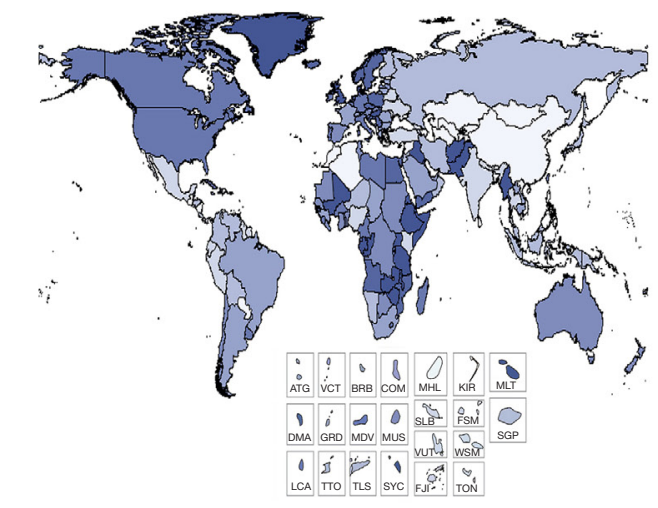

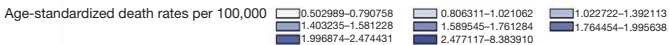

Figure 3 Global and regional bladder cancer ASDR by geography and gender, 1990 and 2016. ASDR, age-standardized death rate; ATG indicates Antigua and Barbuda; VCT, Saint Vincent and the Grenadines; BRB, Barbados; COM, Comoros; MHL, Marshall Islands; KIR, Kiribati; MLT, Malta; DMA, Dominica; GRD, Grenada; MDV, Maldives; MUS, Mauritius; SLB, Solomon Islands; FSM, Federated States of Micronesia; VUT, Vanuatu; WSM, Samoa. SGP, Singapore; LCA, Saint Lucia; TTO, Trinidad and Tobago; TLS, Timor-Leste; SYC, Seychelles; FJI, Fiji; TON, Tonga. 
Table 3 Probability of developing bladder cancer within selected age intervals, globally, and by SDI quintile, by sex, 2006-2016 in \% (odds)

\begin{tabular}{|c|c|c|c|c|c|c|c|c|c|c|c|c|}
\hline \multirow{2}{*}{$\begin{array}{l}\text { Location/ } \\
\text { SDI quintile- }\end{array}$} & \multicolumn{2}{|c|}{ Birth to age 49} & \multicolumn{2}{|c|}{ Age 50 to 59} & \multicolumn{2}{|c|}{ Age 60 to 69} & \multicolumn{2}{|c|}{ Age 70 to 79} & \multicolumn{2}{|c|}{ Age 30 to 70} & \multicolumn{2}{|c|}{ Birth to age 79} \\
\hline & Male & Female & Male & Female & Male & Female & Male & Female & Male & Female & Male & Female \\
\hline Global & $\begin{array}{c}0.05 \\
(1 \text { in } 1,961)\end{array}$ & $\begin{array}{c}0.02 \\
(1 \text { in } 5,319)\end{array}$ & $\begin{array}{c}0.14 \\
(1 \text { in } 740)\end{array}$ & $\begin{array}{c}0.04 \\
(1 \text { in } 2,679)\end{array}$ & $\begin{array}{c}0.38 \\
(1 \text { in } 266)\end{array}$ & $\begin{array}{c}0.09 \\
(1 \text { in } 1,169)\end{array}$ & $\begin{array}{c}0.77 \\
(1 \text { in } 130)\end{array}$ & $\begin{array}{c}0.18 \\
(1 \text { in } 561)\end{array}$ & $\begin{array}{c}1.32 \\
(1 \text { in } 76)\end{array}$ & $\begin{array}{c}0.32 \\
(1 \text { in } 315)\end{array}$ & $\begin{array}{c}1.33 \\
(1 \text { in } 75)\end{array}$ & $\begin{array}{c}0.32 \\
(1 \text { in } 313)\end{array}$ \\
\hline $\begin{array}{l}\text { High- } \\
\text { middle SDI }\end{array}$ & $\begin{array}{c}0.06 \\
(1 \text { in } 1,588)\end{array}$ & $\begin{array}{c}0.02 \\
(1 \text { in } 4,868)\end{array}$ & $\begin{array}{c}0.18 \\
(1 \mathrm{in} 542)\end{array}$ & $\begin{array}{c}0.04 \\
(1 \text { in } 2,558)\end{array}$ & $\begin{array}{c}0.52 \\
(1 \text { in 193) }\end{array}$ & $\begin{array}{c}0.09 \\
(1 \text { in } 1,135)\end{array}$ & $\begin{array}{c}0.98 \\
(1 \text { in 103) }\end{array}$ & $\begin{array}{c}0.18 \\
(1 \text { in } 568)\end{array}$ & $\begin{array}{c}1.73 \\
(1 \text { in } 58)\end{array}$ & $\begin{array}{c}0.32 \\
(1 \mathrm{in} 311)\end{array}$ & $\begin{array}{c}1.73 \\
(1 \text { in } 58)\end{array}$ & $\begin{array}{c}0.32 \\
(1 \text { in } 309)\end{array}$ \\
\hline High SDI & $\begin{array}{c}0.08 \\
(1 \text { in } 1,227)\end{array}$ & $\begin{array}{c}0.04 \\
(1 \text { in } 2,800)\end{array}$ & $\begin{array}{c}0.27 \\
(1 \mathrm{in} 375)\end{array}$ & $\begin{array}{c}0.07 \\
(1 \text { in } 1,388)\end{array}$ & $\begin{array}{c}0.71 \\
(1 \text { in } 140)\end{array}$ & $\begin{array}{c}0.16 \\
(1 \text { in } 618)\end{array}$ & $\begin{array}{c}1.39 \\
(1 \text { in } 72)\end{array}$ & $\begin{array}{c}0.32 \\
(1 \text { in } 316)\end{array}$ & $\begin{array}{c}2.43 \\
(1 \text { in } 41)\end{array}$ & $\begin{array}{c}0.58 \\
(1 \text { in } 172)\end{array}$ & $\begin{array}{c}2.44 \\
(1 \text { in } 41)\end{array}$ & $\begin{array}{c}0.58 \\
(1 \text { in 171) }\end{array}$ \\
\hline $\begin{array}{l}\text { Low- } \\
\text { middle SDI }\end{array}$ & $\begin{array}{c}0.03 \\
(1 \text { in } 3,478)\end{array}$ & $\begin{array}{c}0.01 \\
(1 \text { in } 8,843)\end{array}$ & $\begin{array}{c}0.06 \\
(1 \text { in } 1,621)\end{array}$ & $\begin{array}{c}0.02 \\
(1 \text { in } 4,529)\end{array}$ & $\begin{array}{c}0.15 \\
(1 \text { in } 646)\end{array}$ & $\begin{array}{c}0.05 \\
(1 \text { in } 2,066)\end{array}$ & $\begin{array}{c}0.26 \\
(1 \text { in } 377)\end{array}$ & $\begin{array}{c}0.08 \\
(1 \text { in } 1,206)\end{array}$ & $\begin{array}{c}0.51 \\
(1 \text { in 197) }\end{array}$ & $\begin{array}{c}0.16 \\
(1 \text { in } 611)\end{array}$ & $\begin{array}{c}0.51 \\
(1 \text { in 196) }\end{array}$ & $\begin{array}{c}0.16 \\
(1 \text { in } 608)\end{array}$ \\
\hline Low SDI & $\begin{array}{c}0.03 \\
(1 \text { in } 3,864)\end{array}$ & $\begin{array}{c}0.02 \\
(1 \text { in } 6,406)\end{array}$ & $\begin{array}{c}0.05 \\
(1 \text { in } 2,020)\end{array}$ & $\begin{array}{c}0.03 \\
(1 \text { in } 3,135)\end{array}$ & $\begin{array}{c}0.13 \\
(1 \text { in } 787)\end{array}$ & $\begin{array}{c}0.07 \\
(1 \text { in } 1,453)\end{array}$ & $\begin{array}{c}0.26 \\
(1 \text { in } 385)\end{array}$ & $\begin{array}{c}0.10 \\
(1 \text { in } 964)\end{array}$ & $\begin{array}{c}0.46 \\
(1 \text { in } 217)\end{array}$ & $\begin{array}{c}0.22 \\
(1 \text { in } 457)\end{array}$ & $\begin{array}{c}0.46 \\
(1 \text { in } 217)\end{array}$ & $\begin{array}{c}0.22 \\
(1 \text { in } 455)\end{array}$ \\
\hline Middle SDI & $\begin{array}{c}0.05 \\
(1 \text { in } 1,885)\end{array}$ & $\begin{array}{c}0.02 \\
(1 \text { in } 5,801)\end{array}$ & $\begin{array}{c}0.10 \\
(1 \text { in } 1,034)\end{array}$ & $\begin{array}{c}0.03 \\
(1 \text { in } 3,640)\end{array}$ & $\begin{array}{c}0.24 \\
(1 \text { in } 422)\end{array}$ & $\begin{array}{c}0.05 \\
(1 \text { in } 1,855)\end{array}$ & $\begin{array}{c}0.43 \\
(1 \text { in } 232)\end{array}$ & $\begin{array}{c}0.11 \\
(1 \mathrm{in} 927)\end{array}$ & $\begin{array}{c}0.81 \\
(1 \text { in 123) }\end{array}$ & $\begin{array}{c}0.20 \\
(1 \text { in } 490)\end{array}$ & $\begin{array}{c}0.82 \\
(1 \text { in 122) }\end{array}$ & $\begin{array}{c}0.21 \\
(1 \text { in } 485)\end{array}$ \\
\hline
\end{tabular}

Data in the parentheses indicates 95\% uncertainty interval (95\% UI). SDI, sociodemographic index (a summary indicator of income per capita, educational attainment, and fertility).
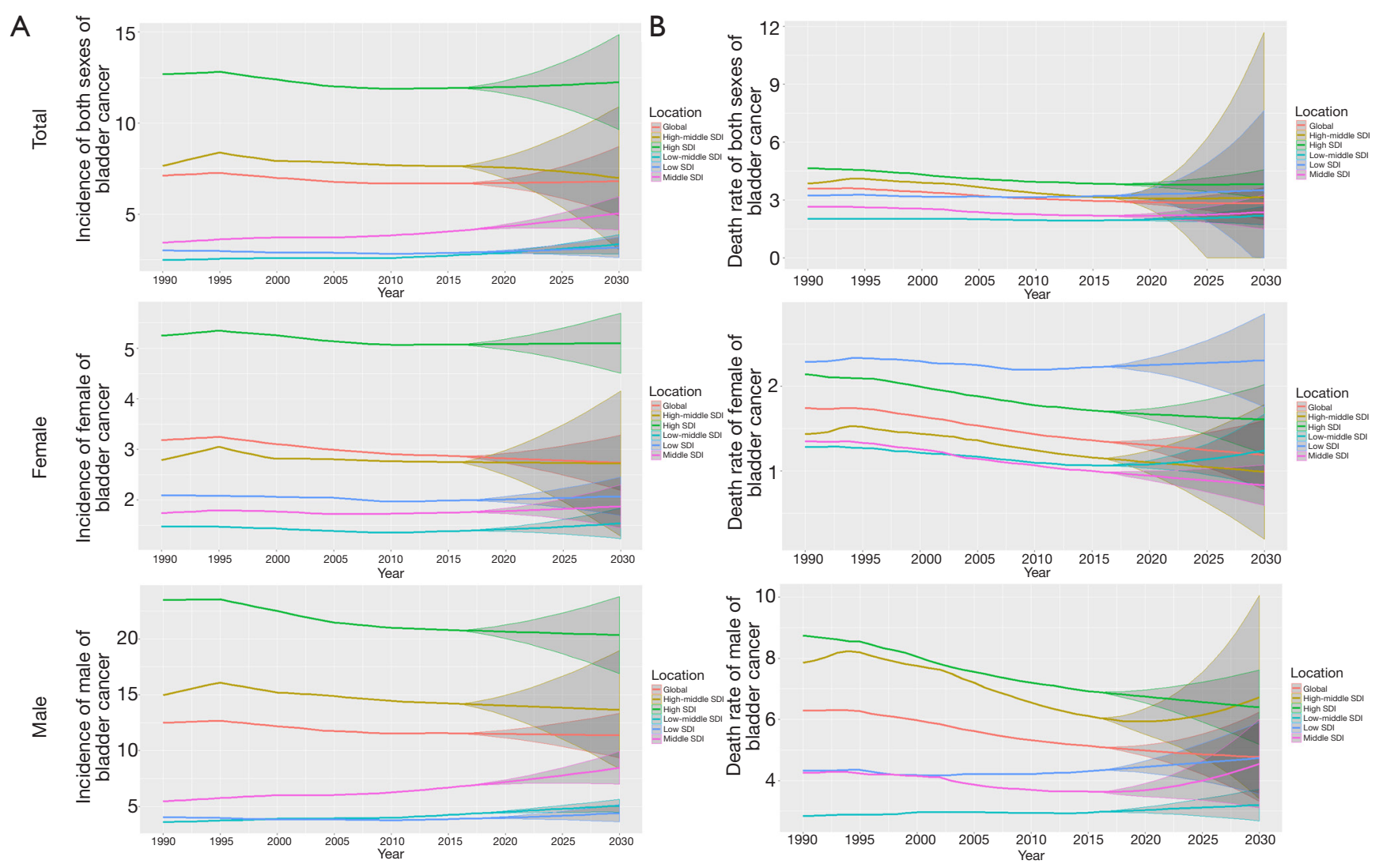

Figure 4 Global and regional trends and predictions in age-standardized incidence and death rates for bladder cancer by SDI quintile, 1990-2030. (A) Trends and predictions in age-standardized incidence rates for bladder cancer by SDI quintile, 1990-2030; (B) trends and predictions in age-standardized death rates for bladder cancer by SDI quintile, 1990-2030. SDI, Sociodemographic index (a summary indicator of income per capita, educational attainment, and fertility). 
prevention and control strategies. However, although we extracted the data accurately, there is likely a small amount of bias in the prediction results. However, future trends in bladder cancer are worthy of attention.

\section{Conclusions}

Globally, between 1990 and 2016, the incidence and death cases of bladder cancer increased substantially, while the ASIR and ASDR of bladder cancer decreased. The incidence and mortality rates of bladder cancer will continue to increase among some SDI countries and regions in the next 15 years. Policy makers in different regions should devise improved strategies for bladder cancer prevention and control.

\section{Acknowledgments}

We thank the Global Burden of Disease Study for collection of the GBD data.

Funding: This study was funded by the National Natural Science Foundation of China (No. 91746205), IBM Global University Programs (2018 IBM Shared University Research Award), Natural Science Foundation of Tianjin (No. 17JCYBJC26000), Tianjin Technical Expert Project and Hospital Innovation \& Management Research Project of Tianjin Medical University (No. 2019YG08).

\section{Footnote}

Conflicts of Interest: All authors have completed the ICMJE uniform disclosure form (available at http://dx.doi. org/10.21037/tau.2020.02.24). The authors have no conflicts of interest to declare.

Ethical Statement: The authors are accountable for all aspects of the work in ensuring that questions related to the accuracy or integrity of any part of the work are appropriately investigated and resolved.

Open Access Statement: This is an Open Access article distributed in accordance with the Creative Commons Attribution-NonCommercial-NoDerivs 4.0 International License (CC BY-NC-ND 4.0), which permits the noncommercial replication and distribution of the article with the strict proviso that no changes or edits are made and the original work is properly cited (including links to both the formal publication through the relevant DOI and the license). See: https://creativecommons.org/licenses/by-nc$\mathrm{nd} / 4.0 /$.

\section{References}

1. Fitzmaurice C, Akinyemiju TF, Al Lami FH, et al. Global, Regional, and National Cancer Incidence, Mortality, Years of Life Lost, Years Lived With Disability, and DisabilityAdjusted Life-Years for 29 Cancer Groups, 1990 to 2016: A Systematic Analysis for the Global Burden of Disease Study. JAMA Oncol 2018;4:1553-68.

2. Siegel RL, Miller KD, Jemal A. Cancer statistics, 2018. CA Cancer J Clin 2018;68:7-30.

3. Heney NM, Ahmed S, Flanagan MJ, et al. Superficial bladder cancer: progression and recurrence. J Urol 1983;130:1083.

4. Chavan S, Bray F, Lortet-Tieulent J, et al. International variations in bladder cancer incidence and mortality. Eur Urol 2014;66:59-73.

5. Antoni S, Ferlay J, Soerjomataram I, et al. Bladder cancer incidence and mortality: a global overview and recent trends. Eur Urol 2017;71:96-108.

6. Fitzmaurice C, Allen C, Barber RM, et al. Global Burden of Disease Cancer Collaboration. Global, regional, and national cancer incidence, mortality, years of life lost, years lived with disability, and disability-adjusted life-years for 32 cancer groups, 1990 to 2015: a systematic analysis for the Global Burden of Disease Study. JAMA Oncol 2017;3:524-48.

7. GBD 2016 Causes of Death Collaborators. Global, regional, and national age-sex specific mortality for 264 causes of death, 1980-2016: a systematic analysis for the Global Burden of Disease Study 2016. Lancet 2017;390:1151-210.

8. GBD 2016 DALYs and HALE Collaborators. Global, regional, and national disability-adjusted life-years (DALYs) for 333 diseases and injuries and healthy life expectancy (HALE) for 195 countries and territories, 1990-2016: a systematic analysis for the Global Burden of Disease Study 2016. Lancet 2017;390:1260-344.

9. Global Burden of Disease Cancer Collaboration, Fitzmaurice C, Dicker D, et al. The Global Burden of Cancer 2013. JAMA Oncol 2015;1:505-27.

10. Forouzanfar MH, Alexander L, Anderson HR, et al. Global, regional, and national comparative risk assessment of 79 behavioral, environmental and occupational, and metabolic risks or clusters of risks in 188 countries, 19902013: a systematic analysis for the Global Burden of 
Disease Study 2013. Lancet 2015;386:2287-323.

11. GBD 2013 Mortality and Causes of Death Collaborators. Global, regional, and national age-sex specific all-cause and cause-specific mortality for 240 causes of death, 19902013: a systematic analysis for the Global Burden of Disease Study 2013. Lancet 2015;385:117-71.

12. Global Burden of Disease Study 2013 Collaborators. Global, regional, and national incidence, prevalence, and years lived with disability for 301 acute and chronic diseases and injuries in 188 countries, 1990-2013: a systematic analysis for the Global Burden of Disease Study 2013. Lancet 2015;386:743-800.

13. Stevens GA, Alkema L, Black RE, et al. GATHER Working Group. Guidelines for Accurate and Transparent Health Estimates Reporting: the GATHER statement. PLoS Med 2016;13:e1002056.

14. GBD 2016 Disease and Injury Incidence and Prevalence Collaborators. Global, regional, and national incidence, prevalence, and years lived with disability for 328 diseases and injuries for 195 countries, 1990-2016: a systematic analysis for the Global Burden of Disease Study 2016. Lancet 2017;390:1211-59.

15. GBD 2016 Risk Factors Collaborators. Global, regional, and national comparative risk assessment of 84 behavioral, environmental and occupational, and metabolic risks or clusters of risks, 1990-2016: a systematic analysis for the Global Burden of Disease Study 2016. Lancet 2017;390:1345-422.

Cite this article as: Cai Q, Chen Y, Xin S, Zhang D, Pan J, Xie Z, Xu C, Li S, Zhang X, Gao Y, Hou J, Guo X, Zhou X, Zhang B, Ma F, Zhang W, Lin G, Xin Z, Niu Y, Wang Y. Temporal trends of bladder cancer incidence and mortality from 1990 to 2016 and projections to 2030. Transl Androl Urol 2020;9(2):153-165. doi: 10.21037/tau.2020.02.24
16. Coleman MP, Quaresma M, Berrino F, et al. Cancer survival in five continents: a worldwide population-based study (CONCORD). Lancet Oncol 2008;9:730-56.

17. GBD 2016 Mortality Collaborators. Global, regional, and national under-5 mortality, adult mortality, age-specific mortality, and life expectancy, 1970-2016: a systematic analysis for the Global Burden of Disease Study 2016. Lancet 2017;390:1084-150.

18. Kaufman DS, Shipley WU, Feldman AS. Bladder cancer. Lancet 2009;374:239-49.

19. Freedman ND, Silverman DT, Hollenbeck AR, et al. Association between smoking and risk of bladder cancer among men and women. JAMA 2011;306:737-45.

20. Burger M, Catto JW, Dalbagni G, et al. Epidemiology and risk factors of urothelial bladder cancer. Eur Urol 2013;63:234-41.

21. Malmström PU, Sylvester RJ, Crawford DE, et al. An individual patient data meta-analysis of the long-term outcome of randomised studies comparing intravesical mitomycin C versus bacillus Calmette-Guerin for nonmuscle-invasive bladder cancer. Eur Urol 2009;56:247-56.

22. Stewart BW, Wild CP. World Cancer Report 2014. World Health Organization. 2014:Chapter 1.1.ISBN9283204298.

23. Yoshino H, Enokida H, Chiyomaru T, et al. Tumor suppressive microRNA-1 mediated novel apoptosis pathways through direct inhibition of splicing factor serine/arginine-rich 9 (SRSF9/SRp30c) in bladder cancer. Biochem Biophys Res Commun 2012;417:588-93. 

Table S2 Number of site-years for bladder cancer mortality data

\begin{tabular}{|c|c|c|c|c|c|c|c|c|c|c|c|c|}
\hline Cause & $\begin{array}{c}\text { VR GBD } \\
2015\end{array}$ & $\begin{array}{c}\text { VR GBD } \\
2016\end{array}$ & $\begin{array}{l}\text { VR change } \\
\text { GBD } 2015 \text { to } \\
\text { GBD } 2016\end{array}$ & $\begin{array}{c}\text { VA GBD } \\
2015\end{array}$ & $\begin{array}{l}\text { VA GBD } \\
2016\end{array}$ & $\begin{array}{l}\text { VA change } \\
\text { GBD } 2015 \text { to } \\
\text { GBD } 2016\end{array}$ & $\begin{array}{c}\text { CR GBD } \\
2015\end{array}$ & $\begin{array}{l}\text { CR GBD } \\
2016\end{array}$ & $\begin{array}{l}\text { CR change } \\
\text { GBD } 2015 \text { to } \\
\text { GBD } 2016\end{array}$ & $\begin{array}{l}\text { Total } \\
\text { GBD } \\
2015\end{array}$ & $\begin{array}{l}\text { Total } \\
\text { GBD } \\
2016\end{array}$ & $\begin{array}{c}\text { Total change } \\
\text { GBD } 2015 \text { to } \\
\text { GBD } 2016\end{array}$ \\
\hline
\end{tabular}

GBD, Global Burden of Disease; VR, vital registration system data; VA, verbal autopsy data; CR, cancer registry data. 


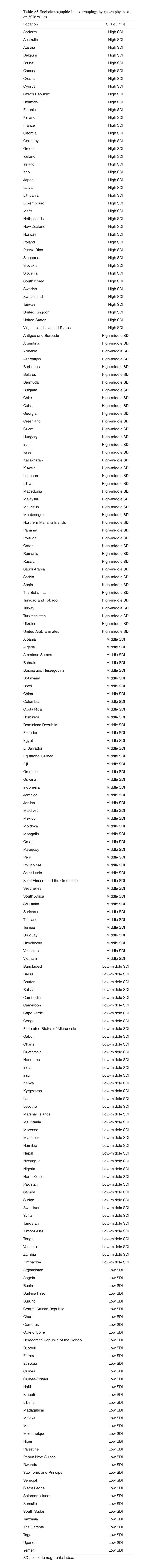


Table S4 Covariates selected for CODEm for each GBD bladder cancer group and expected direction of covariate

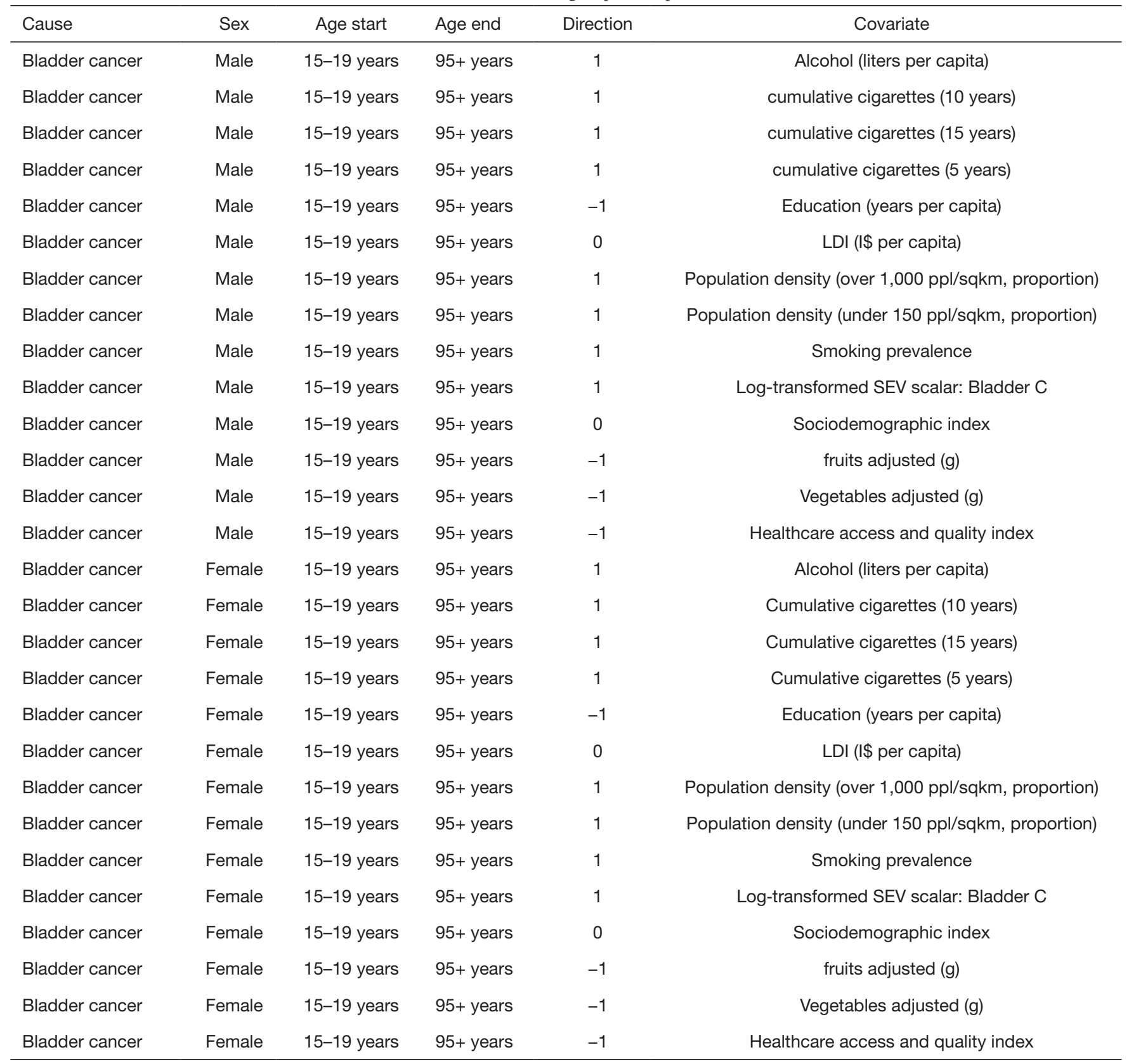

CODEm, cause of death ensemble model; GBD, Global Burden of Disease. 
Table S5 Comparison of GBD 2015 and GBD 2016 covariates used and level of covariates

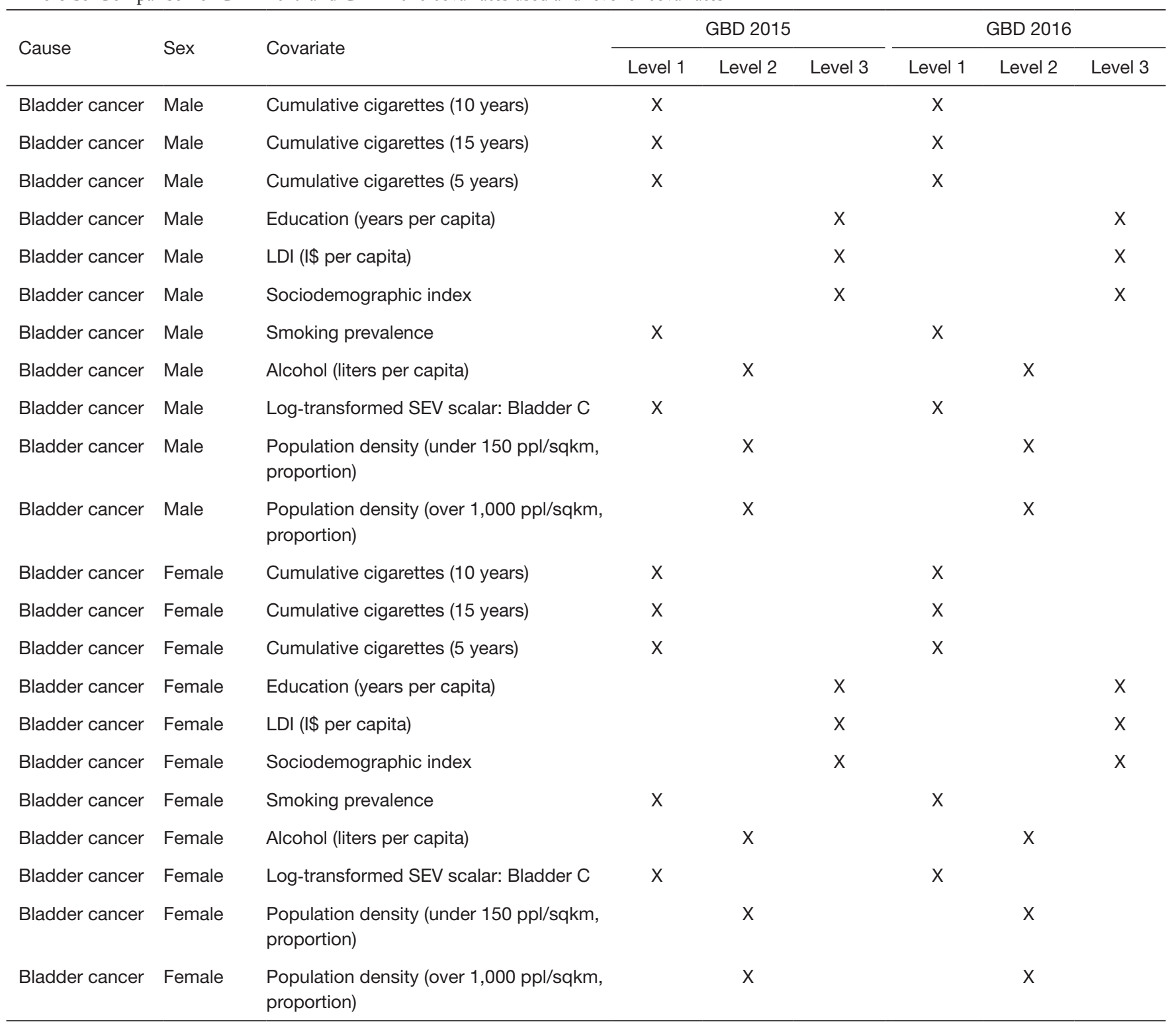

GBD, Global Burden of Disease. 
Table S6 Results for CODEm model testing

\begin{tabular}{|c|c|c|c|c|c|c|c|c|c|}
\hline \multirow{2}{*}{ Cause } & \multirow{2}{*}{ Sex } & \multirow{2}{*}{ Age start } & \multirow{2}{*}{ Age end } & \multicolumn{6}{|c|}{ Predictive validity } \\
\hline & & & & RMSE in & RMSE out & Trend in & Trend out & Coverage in & Coverage out \\
\hline Bladder cancer (data rich) & Male & $15-19$ years & $95+$ years & 0.238162 & 0.289199 & 0.193221 & 0.21405 & 0.998893 & 0.9979 \\
\hline Bladder cancer (global) & Male & $15-19$ years & $95+$ years & 0.259512 & 0.358356 & 0.20438 & 0.211875 & 0.998849 & 0.993972 \\
\hline Bladder cancer (global) & Female & $15-19$ years & $95+$ years & 0.279362 & 0.406151 & 0.217047 & 0.218843 & 0.995797 & 0.992228 \\
\hline Bladder cancer (data rich) & Female & $15-19$ years & $95+$ years & 0.241778 & 0.308128 & 0.197425 & 0.236098 & 0.995139 & 0.99458 \\
\hline
\end{tabular}

CODEm, cause of death ensemble model; RMSE, root mean square of errors.

Table S7 Percent change before and after Cod Correct by bladder cancer for all ages, both sexes combined, 2016

\begin{tabular}{|c|c|c|}
\hline Cause & Cod Correct level & Percent change (\%) \\
\hline Bladder cancer & 3 & $-2.19(-3.56$ to -0.52$)$ \\
\hline
\end{tabular}

Table S8 Disability weights

\begin{tabular}{|c|c|c|c|}
\hline Health state & Lay description & Estimate & Uncertainty interval \\
\hline Cancer, diagnosis and primary therapy & Has pain, nausea, fatigue, weight loss and high anxiety & 0.288 & $0.193-0.399$ \\
\hline Cancer, controlled phase & $\begin{array}{l}\text { Has a chronic disease that requires medication every day and causes some worry but } \\
\text { minimal interference with daily activities }\end{array}$ & 0.049 & $0.031-0.072$ \\
\hline Cancer, metastatic & Has severe pain, extreme fatigue, weight loss and high anxiety & 0.451 & $0.307-0.600$ \\
\hline Terminal phase, with medication & $\begin{array}{l}\text { Has lost a lot of weight and regularly uses strong medication to avoid constant pain. The } \\
\text { person has no appetite, feels nauseous, and needs to spend most of the day in bed }\end{array}$ & 0.540 & $0.377-0.687$ \\
\hline
\end{tabular}

Table S9 Decomposition analysis of bladder cancer incidence trends at the global and regional levels, and by SDI quintiles, both sexes, 2006 to 2016

\begin{tabular}{|c|c|c|c|c|c|c|c|c|}
\hline \multirow[b]{2}{*}{ Location } & \multicolumn{2}{|c|}{ Incidence cases, No. } & \multicolumn{2}{|c|}{ Expected incidence cases, 2016, No. } & \multicolumn{3}{|c|}{ Change in incidence cases, 2006 to 2016, \% } & \multirow{2}{*}{$\begin{array}{l}\text { Overall } \\
\text { change, } \%\end{array}$} \\
\hline & 2006 & 2016 & $\begin{array}{l}\text { Given population } \\
\text { growth alone }\end{array}$ & $\begin{array}{l}\text { Given population } \\
\text { growth and aging }\end{array}$ & $\begin{array}{l}\text { Due to population } \\
\text { growth }\end{array}$ & $\begin{array}{l}\text { Due to change in } \\
\text { age structure }\end{array}$ & $\begin{array}{l}\text { Due to change in } \\
\text { incidence rate }\end{array}$ & \\
\hline Global & $333,876(329,918$ to 337,714$)$ & $437,442(426,709$ to 447,912$)$ & 375,373 & 441,586 & 12.4 & 19.8 & -1.2 & 31 \\
\hline High SDI & $171,627(169,324$ to 173,991$)$ & $213,500(206,159$ to 220,038$)$ & 180,794 & 213,267 & 5.3 & 18.9 & 0.1 & 24.4 \\
\hline High-middle SDI & $76,815(75,044$ to 78,668$)$ & $96,865(93,605$ to 100,135$)$ & 85,342 & 99,392 & 11.1 & 18.3 & -3.3 & 26.1 \\
\hline Middle SDI & $56,859(55,417$ to 58,408$)$ & $85,371(80,381$ to 87,836$)$ & 61,034 & 77,034 & 7.3 & 28.1 & 14.7 & 50.1 \\
\hline Low-middle SDI & $23,940(22,710$ to 25,170$)$ & $34,771(32,913$ to 36,384$)$ & 27,918 & 32,388 & 16.6 & 18.7 & 10 & 45.2 \\
\hline Low SDI & $6,011(5,650$ to 6,672$)$ & $8,223(7,850$ to 8,585$)$ & 7,952 & 8,197 & 32.3 & 4.1 & 0.4 & 36.8 \\
\hline
\end{tabular}

SDI, sociodemographic index. 